\title{
Prevalence of Scapular Dyskinesis in Breastfeeding Postnatal Women in Satara District - An Observational Study
}

\author{
Gandhali Atul Situt ${ }^{1}$, Shyma Philip², Poonam Patil ${ }^{3}$ \\ ${ }^{1,2}$ Department of Physiotherapy, Krishna Institute of Medical Sciences, Malkapur, Maharashtra, India. ${ }^{3}$ Department \\ of Cardiopulmonary Sciences, Krishna Institute of Medical Sciences, Malkapur, Maharashtra, India
}

\section{ABSTRACT}

\section{BACKGROUND}

Poor posture like hunching forward is one of the most commonly practiced postures by women during breastfeeding. The maintenance of such postures for prolonged period can result in progressive weakness of the scapular muscles and subsequent increase in the kyphotic curve. Both lordosis and kyphosis increase during pregnancy and are observed to be maintained for up to 2 months postnatally. The thoracic spine position and slouched position significantly affects the scapular dynamics during scapular abduction resulting in decreased muscle forces. Thus, scapular dyskinesis, defined as an alteration of normal position or motion of the scapula during coupled scapulohumeral movements can occur as a result of decreased muscle forces. Thus, this gives rise to the need for evaluating scapular dyskinesis and its prevalence in postnatal women. The purpose of the study was to find the prevalence of scapular dyskinesis in breastfeeding postnatal women.

\section{METHODS}

An observational analytical study was undertaken at Krishna Institute of Medical Sciences, including a total of 40 subjects within the age group of $(15-40)$ yrs. who had been breastfeeding for 6 months or more. These subjects were assessed for scapular dyskinesis using Yes / No test and the lateral scapular slide test (LSST). Kyphosis was assessed on observational basis through postural assessment in these individuals. Statistical analysis was done using the InStat app.

\section{RESULTS}

$67 \%$ and $75 \%$ of the subjects were found to be positive for scapular dyskinesis through Yes / No Test and LSST test respectively. Observational assessment showed that $55 \%$ of the subjects were positive for kyphosis. Subjects demonstrated significant prevalence of scapular dyskinesis and subsequent kyphosis in postnatal females due to wrong ergonomic practice of breastfeeding.

\section{CONCLUSIONS}

The study shows that women lack proper knowledge about the breastfeeding ergonomics leading to weakness of scapular muscles. Thus, a significant prevalence of scapular dyskinesis was seen in breastfeeding postnatal women.

\section{KEY WORDS}

Breastfeeding, Scapular Muscle Weakness, Kyphosis, Ergonomics, Scapular Dyskinesis
Corresponding Author: Dr. Gandhali Atul Situt, Final Year B. P. Th,

Krishna Institute of Medical Sciences, Malkapur, Karad City, Satara District, Maharashtra, India.

E-mail: gandhusitut@gmail.com

DOI::14260/jemds/2021/492

How to Cite This Article:

Situt GA, Philip S, Patil P. Prevalence of scapular dyskinesis in breastfeeding postnatal women in Satara district - an observational study. J Evolution Med Dent Sci 2021;10(31):2401-2405, DOI: $10.14260 /$ jemds/2021/492

Submission 19-04-2021,

Peer Review 03-07-2021,

Acceptance 10-07-2021,

Published 02-08-2021.

Copyright (C) 2021 Gandhali Atul Situt et al. This is an open access article distributed under Creative Commons Attribution License [Attribution 4.0 International (CC BY 4.0)] 


\section{BACKGROUND}

Pregnancy and postnatal periods are when the women may experience many physiological and anatomical changes, particularly postural changes, in their body. To maintain the centre of gravity within the base of support during pregnancy postural changes occur consequently affecting the thoracic spine, which is necessary to maintain balance and stability. ${ }^{1}$ The complaints during pregnancy can also continue postpartum. Analysis of a study revealed that both lordosis and kyphosis increased during pregnancy, and that these spinal curvatures were maintained for up to two months postnatally. ${ }^{2}$

Poor posture is most commonly maintained by women during breastfeeding in the postnatal period which results in micro trauma or fatigue causing pain. ${ }^{3}$ Women generally tend to hunch forward during breastfeeding, if this posture is maintained even for 20 minutes, 6 - 8 times or more per day can lead to stress on the back, neck and shoulders. Increase in thoracic flexion can affect the scapulohumeral relationship as this posture affects the scapular kinematics which in turn results in decreased scapular plane shoulder abduction range of motion and muscle force. ${ }^{4}$ Thus, the slouched posture can cause shoulder complex muscle weakness and limit the glenohumeral joint range of motion and can also cause shoulder pain, increased scapular superior translation is also known to occur between neutral and $90^{\circ}$ of arm abduction which may place the upper trapezius muscle in a shortened position and this may thus decrease its ability to generate tension. $.6,7$ This gives rise to the need of evaluating the upper quadrant musculoskeletal involvement during the post birth period and its relation with changes occurring during pregnancy. Thus, the posture during pregnancy and breastfeeding may cause increased kyphotic curve of the thoracic spine. This kyphotic posture if remained uncorrected leads to weakness and fatigue of scapular musculature, tightness of pectoral muscles and back pain. Thus, to study scapular stability is imperative in postpartum females. ${ }^{3}$

Scapular dyskinesia or scapular dysfunction is not an injury or a musculoskeletal diagnosis but rather an alteration of the normal position or motion of scapula during coupled scapulohumeral movements. 'Dys' (alteration of) 'kinesis' (motion) is a general term that reflects loss of normal static and dynamic control of scapular motion. It is a collective term that refers to the movement of scapula that is dysfunction. 8,9

This dysfunction is divided into four movement patterns: 1) abnormal static scapular position and / or dynamic scapular motion characterized by medial border prominence; or 2) inferior angle prominence and / or early scapular elevation or shrugging on arm elevation; or 3) rapid downward rotation during arm lowering; 4) both scapulae rotate symmetrically upward with the inferior angles rotating laterally away from the midline. ${ }^{10,11} \mathrm{~A}$ large number of factors cause dyskinesis; these include: 1) bone causes like kyphosis 2) joint causes like glenohumeral internal derangement 3) neurological causes like long thoracic or spinal accessary nerve palsy, subscapular nerve entrapment 4) soft tissue causes like inflexibility or stiffness of pectoralis minor and short head of biceps, intrinsic muscle pathology, glenohumeral internal rotation deficit which results in winging of scapula. ${ }^{8}$
Normal scapulohumeral rhythm, the coordinated movement of the scapula and humerus to achieve shoulder motion, is the key to efficient shoulder function, thus the scapular plane abduction occurs as an integrated movement at glenohumeral and scapulothoracic joints which is known as scapulohumeral rhythm.1,5 As the spinal alignment affects the scapular position and both of these affect the shoulder girdle function, which mainly occurs because of the numerous muscular connections between the spine, scapula, clavicle and humerus, thus the change of position in any of these bony segments will also change the other ${ }^{5,12}$ If the scapula acts as the stable base during the scapular plane abduction then the glenohumeral $(\mathrm{GH})$ joint will be able to move through its full range of motion.5,13,14 Any condition causing trapezius or rhomboid muscle weakness, multidirectional instability, or any lesion to long thoracic nerve resulting in serratus anterior or trapezius palsy, voluntary action or a painful shoulder resulting in splinting of $\mathrm{GH}$ joint, which in turn causes reverse scapulohumeral rhythm. ${ }^{10}$

A research stated that subjects with kyphosis and rounded shoulders had increased incidence of cervical, inter scapular pain. Scapular stabilization requires coupling of the upper trapezius, middle trapezius, lower trapezius and rhomboid muscles along with serratus anterior muscle. ${ }^{3}$ In kyphosis there is weakness of scapular control muscles and they cannot counteract the altered scapulohumeral rhythm which may lead to dyskinesis.

The World Health Organization and UNICEF recommend breastfeeding from the first hour after the birth, exclusively for the first six months; and continued breastfeeding for two years or more. Most women in India breastfeed for at least one year and if this breastfeeding is continued in bad posture for such long durations, it may cause progressive scapular muscle weakness which may thus lead to scapular dyskinesis in the later stages.

\section{Objectives}

1. To find out the prevalence of scapular dyskinesis in postnatal females due to breastfeeding

2. To find the percentage of thoracic kyphosis by observational assessment in postnatal women

3. To find out the percentage of scapular dyskinesis through qualitative assessment (Yes/No test)

4. To find out the percentage of scapular dyskinesis by quantitative measurements (LSST test).

\section{METHODS}

An analytical observational study for the duration of 6 months from November 2019 to May 2020 was conducted at Krishna hospital, outpatient department, Karad, on a sample size of 40 , the sample size was calculated using the formula $n=z^{2} \times \delta^{2} / e^{2}$

Where

$\mathrm{z}=$ standard normal variable at $95 \%$ class interval

$\delta=$ standard deviation LSST for 6 months left side $=1.164$

$\mathrm{e}=$ allowable error $\geq 5 \%$ at $95 \%$ class interval $=0.05$

$\mathrm{n}=$ sample size 
The sample size was calculated using reference of this article, Krishna Kumar A, Dabholkar TA, Dabholkar A. Assessment of Scapular Stability in Postpartum Females: A Longitudinal Study. In Ergonomics in Caring for People 2018 (pp. 91 - 96). Springer, Singapore. Postnatal women who have been breastfeeding for a duration of more than 6 months and within the age group of (15 - 40) yrs. were included who underwent either full-term normal delivery or lower segment caesarean section. Subjects with any pathology or comorbidities causing kyphosis or scapular dyskinesis were excluded from the study. The study was ethically approved by the institutional ethical committee, KIMS (Deemed to Be University), Karad. Subjects were instructed about the details of the procedure. Demographic data like age and duration of breastfeeding were noted. The presence of kyphosis was noted by postural observation along the sagittal plane. The subjects were asked to stand in relaxed position and any deviation from the normal posture like slouching or round back was noted.

Yes / no test was done for qualitative assessment in which the subjects were asked to stand straight with their hands alongside the body and the elbows kept straight besides the body, shoulders relaxed and in the neutral position. The examiner stood behind the subjects and they were asked to hold $1 \mathrm{~kg}$ weight in each hand and then to abduct the shoulders to their maximum. The participants were asked to repeat the same motion 3 times. Prominence of the scapula was noted while the subjects performed the motion and any observation was noted as 'yes' and absence of any prominence was noted as 'no'.15 The type of dyskinesis was obtained according to the type of border prominence which was observed in the test. For the quantitative assessment, lateral scapular slide test [LSST] was performed in three positions. ${ }^{16}$ The subjects were asked to stand in front of the examiner and the test was performed at the following angles $-0^{\circ}, 45^{\circ}, 120^{\circ}$ of shoulder abduction. In the first position the hands were placed beside the body i.e. arms abducted at $0^{\circ}$, in the second position the arms abducted with elbows flexed and hands resting on the waist with the thumb placed behind i.e. at $45^{\circ}$ and in the third position the arms abducted at $120^{\circ}$ and elbows extended. The distance between the base of spine of scapula and the spinous process of T3 and the distance from the inferior angle of scapula to the spinous process of T7 or T9 were measured. These distances were measured on both the left and right sides and in all the three test positions. Any difference in measurements between the left and right sides occurring at any of the three test positions was noted. A difference of 1 to $1.5 \mathrm{~cm}$ or more between the measurements of left side and right side at any of the two levels and at any of the three angles will be noted as positive for the test.

The main outcome measures used were the presence of kyphosis, the positive response to yes / no test and the LSST test.

\section{Statistical Analysis}

The outcome measures were assessed at the baseline. The collected data in this study was statistically analysed using the InStat app. The age and months of breastfeeding were analysed using mean and standard deviation. The kyphosis was assessed on observational basis and analysed by calculation of its percentage. Scapular dyskinesis was qualitatively assessed using the Yes / No test and was analysed using percentage and quantitatively assessed using LSST test and the unpaired t-test values calculated at each of the three test positions.

\section{RESULTS}

Among the 40 subjects $40 \%$ were presented with T2 type of dyskinesis which is prominence of medial border of scapula. $22 \%$ were of T1 type, $16 \%$ were of T3 type and $6 \%$ were presented with $\mathrm{T} 4$ type of dyskinesis.

\begin{tabular}{|ccc|}
\hline Type & Frequency & Percentage \\
T I & 10 & $22 \%$ \\
T II & 15 & $40 \%$ \\
T III & 6 & $16 \%$ \\
T IV & 2 & $6 \%$ \\
T I+ T II & 2 & $5 \%$ \\
T II + T III & 3 & $8 \%$ \\
T I + T III & 1 & $3 \%$ \\
\hline Table 1. Type of Dyskinesis [on the Basis of Yes / No Test]
\end{tabular}

\begin{tabular}{|c|c|c|c|c|c|}
\hline & & & & $\begin{array}{l}\text { Unpaired } \mathrm{t} \text { - Test } \\
\text { Value }\end{array}$ & P - Value \\
\hline \multirow{6}{*}{$\begin{array}{l}\text { Position 1: } \\
\text { Standing }\end{array}$} & At t3 & Left & Right & \multirow{3}{*}{0.05244} & \multirow{3}{*}{0.9583} \\
\hline & Mean & 6.315 & 6.1725 & & \\
\hline & SD & 1.404 & 1.019 & & \\
\hline & At t7 & & & \multirow{3}{*}{0.09728} & \multirow{3}{*}{0.9228} \\
\hline & Mean & 9.1475 & 9.0725 & & \\
\hline & SD & 1.303 & 0.9578 & & \\
\hline \multirow{6}{*}{$\begin{array}{l}\text { Position 2: } 45^{\circ} \\
\text { Abduction }\end{array}$} & At t3 & Left & Right & \multirow{3}{*}{0.05244} & \multirow{3}{*}{0.9583} \\
\hline & Mean & 5.0535 & 5.55 & & \\
\hline & SD & 1.320 & 1.237 & & \\
\hline & At t7 & & & \multirow{3}{*}{0.09728} & \multirow{3}{*}{0.9228} \\
\hline & Mean & 8.6875 & 8.7125 & & \\
\hline & SD & 1.211 & 1.084 & & \\
\hline \multirow{6}{*}{$\begin{array}{l}\text { Position 3: } 120^{\circ} \\
\text { Abduction }\end{array}$} & At t3 & Left & Right & \multirow{3}{*}{0.7230} & \multirow{3}{*}{0.4719} \\
\hline & Mean & 3.855 & 3.635 & & \\
\hline & SD & 1.349 & 1.372 & & \\
\hline & At t7 & & & \multirow{3}{*}{0.2688} & \multirow{3}{*}{0.7888} \\
\hline & Mean & 9.29 & 9.1875 & & \\
\hline & SD & 1.699 & 1.711 & & \\
\hline
\end{tabular}

Unpaired $\mathrm{T}$ - test was performed to compare the difference in measurements. On statistical analysis $P$ value and $\mathrm{T}$ value in standing position at $\mathrm{T} 3$ were 0.9583 and 0.05244 respectively. $P$ value and $t$ value in standing position at T7 were 0.9228 and 0.09728 respectively. At $45^{\circ}$ abduction position at T3 level the $\mathrm{P}$ value and $\mathrm{T}$ value were 0.9583 and 0.05244 respectively. At $45^{\circ}$ abduction position at $\mathrm{T} 7$ level the $P$ value and $t$ value were 0.9228 and 0.09728 respectively. At $120^{\circ}$ of abduction position at T3 level the $\mathrm{P}$ value was 0.4719 and the $\mathrm{T}$ value was 0.7230 and at T7 level the $\mathrm{P}$ value was 0.7888 and $\mathrm{T}$ value was 0.2688 . Therefore, according to the statistical analysis performed prevalence of scapular dyskinesis was found to be significant.

On observation $55 \%$ subjects showed the presence of postural kyphosis, while $45 \%$ were non kyphotic. The qualitative test performed for scapular dyskinesis was the Yes / No Test on the basis of which it was found $67 \%$ of subjects among the total number of 40 samples taken showed positive response for scapular dyskinesis, whereas $33 \%$ of subjects showed negative response for scapular dyskinesis. On the basis of the quantitative test performed i.e., the LSST Test $75 \%$ of the subjects were found to be positive while the remaining $25 \%$ were found to be negative. 

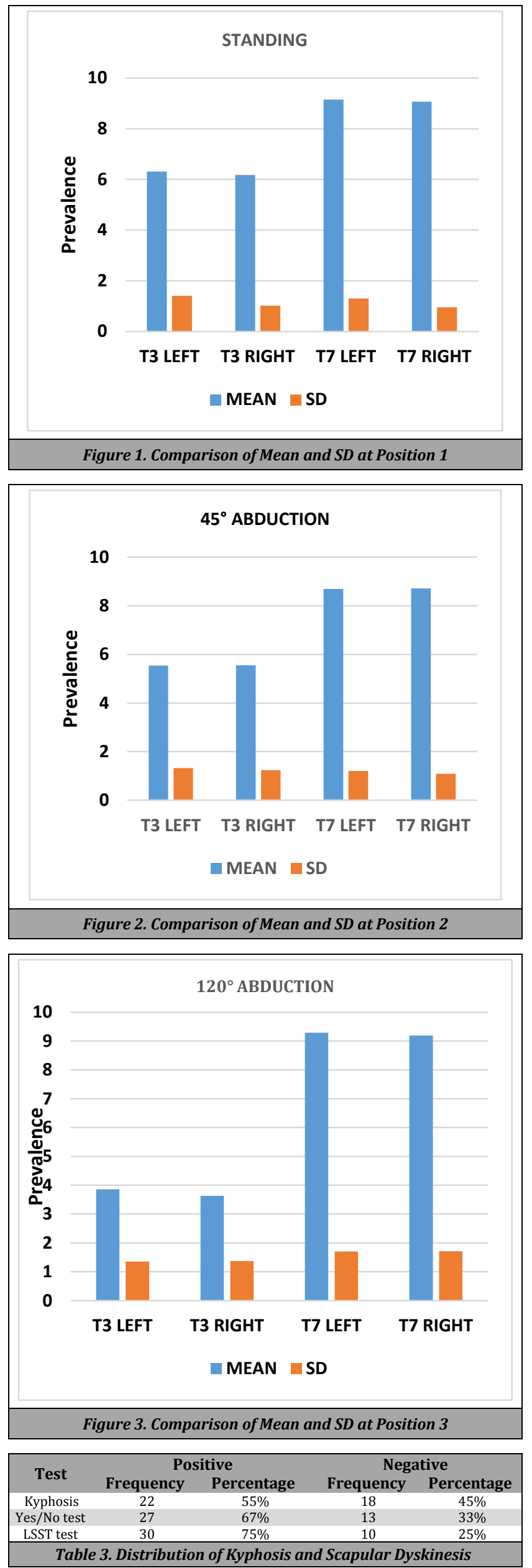

\section{DISCUSSION}

Postpartum females who have undergone either full term normal delivery (FTND) or lower segment caesarean section (LSCS) and were breastfeeding for at least 6 months were included in the study. Females with any pathology or comorbidities causing kyphosis or scapular dyskinesis were not considered.

40 subjects with age group of $15-40$ yrs.were selected. The mean of the age group was 26.2. Even though the age was not considered as a risk factor, the study shows that females at their young age are suffering from kyphosis and scapular dyskinesis. The mean of the duration of breastfeeding showed that those women who breastfed for more than 6 months had a greater prevalence of scapular dyskinesis. A major risk factor thus observed was duration of breastfeeding; as the months of breastfeeding in altered posture increases, the risk of scapular dyskinesis also increases. Such subjects are found to be kyphotic too which shows weakness of scapular muscles. A study by Goyal, Ram $\mathrm{C}$ et al. supports the above findings as the study revealed poorer positioning among primipara $(24.0 \%)$ than multipara (8.9-12.5\%) mothers and parity was seen to be significantly associated with poor position. ${ }^{17}$

Studies by Raikes. A and Kibler WB state that the Yes / No test considers multiple plane asymmetries in clinical assessment and is therefore a good screening tool for assessment of scapular dyskinesis, ${ }^{18,19}$ also a study suggests that mild scapular dyskinesis can frequently be seen during the lowering phase of the arm movement, commonly during eccentric muscle contraction which might be because of the alteration in the neuromuscular control during the contraction and the Yes / No Test showed the same. ${ }^{11}$

The qualitative test (Yes / No test) when compared to the quantitative test (LSST Test) showed that there was a greater prevalence of scapular dyskinesis in the quantitative test performed. This reveals that though the subjects may not be positive for dyskinesis on observational assessment they still might have minor weakness of scapular muscles and altered scapular motion due to the same, which is seen in the quantitative assessment by a difference in the position of scapula on the left and right sides.

A previous study about assessment of scapular stability in postpartum females: a longitudinal study by Akhila Krishnakumar, Tejashree A. Dabholkar and Ajit Dabholkar concluded that postpartum females showed increased tightness of pectoralis minor muscle and decreased strength of rhomboids and lower trapezius. Six outcome measures were used in that study and the positive results also showed statistically significant change of scapular stability. ${ }^{3}$

Many researches have put forward that postural changes during pregnancy is due to shift in the centre of the gravity. ${ }^{1}$ A research analysed that both lordosis and kyphosis increased significantly during pregnancy and these curves were maintained for up to 2 months postnatally. ${ }^{2}$ Also subjects with rounded shoulders and kyphosis had increased incidence of cervical and intrascapular pain. ${ }^{3}$ A study on scapular dyskinesis by WB Kibler has mentioned kyphosis, tightness of upper chest muscles and weakness of upper back muscles as causes for scapular dyskinesis. ${ }^{10}$ 
On the basis of above researches this study focused on evaluating the upper quadrant musculoskeletal involvement during post birth period and showed how significant it is, as according to the results of statistical analysis scapular dyskinesis is seen in weighted shoulder movements and also positive results are found in LSST analysis. This is due to kyphosis or tightness of pectoral muscles and weakness of scapular muscles due to prolonged slouched posture during breastfeeding and shift of centre of gravity of the body.

Scapular dyskinesis was measured and diagnosed on the basis of the gold standard special test LSST on the basis of various studies performed, ${ }^{19}$ however any objective assessment for diagnosing scapular dyskinesis was not used, thus a more reliable method like modified LSST or a device could be used as suggested by the following study done by A Shadmehr. ${ }^{20}$ Additional research is needed on musculoskeletal complications occurring as a result of wrong ergonomic practice during breastfeeding. Objective assessment tool for measuring the weakness of scapular muscles can be used for better accuracy. Number of pregnancies and its correlation with scapular dyskinesis can be evaluated. The study can be performed on a larger population.

\section{CONCLUSIONS}

The study revealed that most of the women lack proper knowledge about the breastfeeding positions and hence tend to practice breastfeeding in slouched and uncomfortable postures, putting stress on their back muscles and weakening them resulting in scapular dyskinesis over time. Thus, a significant prevalence of scapular dyskinesis was found in postnatal women.

\section{Limitations}

The study included a few limitations, the kyphosis in the following subjects was not measured by any objective assessment tool and was done only on observational basis and thus only the presence of it was noted. The study was conducted in a small area on a limited population. The strength of the scapular muscles was not measured.

Data sharing statement provided by the authors is available with the full text of this article at jemds.com.

Financial or other competing interests: None.

Disclosure forms provided by the authors are available with the full text of this article at jemds.com.

\section{REFERENCES}

[1] Okanishi N, Kito N, Akiyama M, et al. Spinal curvature and characteristics of postural change in pregnant women. Acta Obstet Gynecol Scand 2012;91(7):856-61.

[2] Bullock-Saxton JE. Changes in posture associated with pregnancy and the early post-natal period measured in standing. Physiotherapy Theory and Practice 1991;7(2):103-9.

[3] Krishnakumar A, Dabholkar TA, Dabholkar A. Assessment of scapular stability in postpartum females: a longitudinal study. In: Ergonomics in Caring for People. Singapore: Springer 2018:91-6.

[4] Kibler WB, Sciascia AD. Scapular dyskinesis and glenohumeral instability. In Disorders of the Scapula and their Role in Shoulder Injury. Cham: Springer 2017:7989.

[5] Kebaetse M, McClure P, Pratt NA. Thoracic position effect on shoulder range of motion, strength and threedimensional scapular kinematics. Arch Phys Med Rehabil 1999;80(8):945-50.

[6] Esmaeelnezhad M, Ahmadi A, Maroufi N, et al. Comparison of scapular posture between individuals with and without forward head posture using a clinical test. J Rehab Med 2018;7(1):208-15.

[7] Plummer HA, Sum JC, Pozzi F, et al. Observational scapular dyskinesis: known-groups validity in patients with and without shoulder pain. J Orthop Sports Phys Ther 2017;47(8):530-7.

[8] López-Vidriero E, López-Vidriero R, Rosa LF, et al. Scapular dyskinesis: related pathology. International Journal of Orthopaedics 2015;2(1):191-5.

[9] Tate AR, McClure P, Kareha S, et al. A clinical method for identifying scapular dyskinesis, part 2: validity. J Athl Train 2009;44(2):165-73.

[10] Magee DJ, Manske RC. Orthopedic physical assessment. Elsevier Health Sciences 2020.

[11] McClure P, Tate AR, Kareha S, et al. A clinical method for identifying scapular dyskinesis, part 1: reliability. J Athl Train 2009;44(2):160-4.

[12] Thigpen CA, Padua DA, Michener LA, et al. Head and shoulder posture affect scapular mechanics and muscle activity in overhead tasks. J Electromyogr Kinesiol 2010;20(4):701-9.

[13] McClure P, Greenberg E, Kareha S. Evaluation and management of scapular dysfunction. Sports Med Arthrosc Rev 2012;20(1):39-48.

[14] Paine R, Voight ML. The role of the scapula. Int J Sports Physl Ther 2013;8(5):617-29.

[15] Tafreshi ES, Moghadam AN, Bakhshi E, et al. Comparing scapular position and scapular dyskinesis in individuals with and without rounded shoulder posture. Physical Treatments-Specific Physical Therapy Journal 2015;5(3):127-36.

[16] Park SY. Interrater reliability and intrarater reliability of lateral scapular slide tests of females in their 20s. J Phys Ther Sci 2017;29(4):726-8.

[17] Goyal RC, Banginwar AS, Ziyo F, et al. Breastfeeding practices: positioning, attachment (latch-on) and effective suckling-a hospital-based study in Libya. J Fam Community Med 2011;18(2):74-9.

[18] Raikes A. The reliability and diagnostic accuracy of the yes/no scapular dyskinesis test when used by graduate assistant athletic trainers. All Graduate Thesis and Dissertations 2012

[19] Uhl TL, Kibler WB, Gecewich B, et al. Evaluation of clinical assessment methods for scapular dyskinesis. Arthroscopy 2009;25(11):1240-8.

[20] Shadmehr A, Sarafraz H, Blooki MH, et al. Reliability, agreement and diagnostic accuracy of the modified lateral scapular slide test. Manual Therapy 2016;24:1824. 